\title{
Few-cycle solitons and supercontinuum generation with cascaded quadratic nonlinearities in unpoled lithium niobate ridge waveguides
}

\author{
Guo, Hairun; Zeng, Xianglong; Zhou, Binbin; Bache, Morten
}

Published in:

Optics Letters

Link to article, DOI:

10.1364/OL.39.001105

Publication date:

2014

Document Version

Publisher's PDF, also known as Version of record

Link back to DTU Orbit

Citation (APA):

Guo, H., Zeng, X., Zhou, B., \& Bache, M. (2014). Few-cycle solitons and supercontinuum generation with cascaded quadratic nonlinearities in unpoled lithium niobate ridge waveguides. Optics Letters, 39(5), 1105-1108. https://doi.org/10.1364/OL.39.001105

\section{General rights}

Copyright and moral rights for the publications made accessible in the public portal are retained by the authors and/or other copyright owners and it is a condition of accessing publications that users recognise and abide by the legal requirements associated with these rights.

- Users may download and print one copy of any publication from the public portal for the purpose of private study or research.

- You may not further distribute the material or use it for any profit-making activity or commercial gain

- You may freely distribute the URL identifying the publication in the public portal 


\title{
Few-cycle solitons and supercontinuum generation with cascaded quadratic nonlinearities in unpoled lithium niobate ridge waveguides
}

\author{
Hairun Guo, ${ }^{1}$ Xianglong Zeng, ${ }^{1,2,3}$ Binbin Zhou, ${ }^{1}$ and Morten Bache ${ }^{1,4}$ \\ ${ }^{1}$ DTU-Fotonik, Department of Photonics Engineering Technical University of Denmark, DK-2800, Kgs. Lyngby, Denmark \\ ${ }^{2}$ Key Laboratory of Special Fiber Optics and Optical Access Networks, Shanghai University, Shanghai 200072, China \\ ${ }^{3}$ e-mail: zenglong@shu.edu.cn \\ ${ }^{4}$ e-mail: moba@fotonik.dtu.dk
}

Received December 3, 2013; revised January 16, 2014; accepted January 16, 2014; posted January 16, 2014 (Doc. ID 202442); published February 17, 2014

\begin{abstract}
Formation and interaction of few-cycle solitons in a lithium niobate ridge waveguide are numerically investigated. The solitons are created through a cascaded phase-mismatched second-harmonic generation process, which induces a dominant self-defocusing Kerr-like nonlinearity on the pump pulse. The inherent material self-focusing Kerr nonlinearity is overcome over a wide wavelength range, and self-defocusing solitons are supported from 1100 to $1900 \mathrm{~nm}$, covering the whole communication band. Single cycle self-compressed solitons and supercontinuum generation spanning 1.3 octaves are observed when pumped with femtosecond nanojoule pulses at $1550 \mathrm{~nm}$. The waveguide is not periodically poled, as quasi-phase-matching would lead to detrimental nonlinear effects impeding few-cycle soliton formation. (C) 2014 Optical Society of America
\end{abstract}

OCIS codes: (230.7370) Waveguides; (320.5520) Pulse compression; (320.6629) Supercontinuum generation; (320.7110) Ultrafast nonlinear optics.

http://dx.doi.org/10.1364/OL.39.001105

Waveguides in quadratic nonlinear crystals are of great interest because the waveguide will provide strong confinement on the light and suppress the spatial diffraction, which equivalently promotes the efficiency of the nonlinear interaction and therefore pulses with low energy could be operated. With the quadratic nonlinearity giving rise to second-harmonic generation (SHG), or more generally a three-wave mixing (TWM) process, quadratic waveguides find applications in the field of integrated waveguide optics and fiber communications for, e.g., frequency doubling, or frequency upconversions/ downconversions. In most cases the waveguides are prepared for noncritical interactions (so-called type-0 interaction, where all waves in the TWM process are identically polarized), which exploits the largest quadratic tensor components but unfortunately cannot be phase-matched. Quasi-phase-matching (QPM) is therefore used to remove the residual phase mismatch and achieve an effective phase-matching condition. Using QPM extends the wavelength range for sum- and difference-frequency generation [1], and extends the phasematching bandwidth for optical parametric oscillators and amplifiers [2].

On the contrary, in strongly phase-mismatched conditions quadratic nonlinearities produce a so-called cascaded quadratic nonlinearity [3], which acts like a cubic Kerr self-phase modulation (SPM) nonlinearity in inducing an intensity-dependent nonlinear phase shift on the pump pulse. The equivalent Kerr-like nonlinear index is [3] $n_{2, \text { casc }} \propto-d_{\text {eff }}^{2} / \Delta k$, where $d_{\text {eff }}$ is the effective quadratic nonlinearity and the phase mismatch is $\Delta k=$ $k_{2}-2 k_{1}$, where $k_{1}$ and $k_{2}$ are the pump and SH wavenumbers, respectively. This will directly compete with the inherent material self-focusing Kerr nonlinearity $n_{2, \text { Kerr }}>0$, resulting in an effective nonlinear index change $\Delta n=n_{2, \text { eff }} I=\left(n_{2, \text { casc }}+n_{2, \mathrm{Kerr}}\right) I$. When achieving an effective self-defocusing $\left(n_{2, \text { eff }}<0\right)$ nonlinearity, bulk quadratic materials have received much attention for few-cycle pulse compression, soliton formation, and soliton-induced supercontinuum generation (SCG) of energetic femtosecond pulses [4-7], where the absence of self-focusing effects implies that much more energetic pulses can be used than in traditional Kerr media. Similarly, in quadratic waveguides soliton-induced SCG has been demonstrated in lithium niobate (LN) waveguides [8,9] pumped in the near-IR where LN has normal dispersion. Thus, soliton formation is only possible because the effective nonlinearity is self-defocusing due to a dominant negative cascading nonlinearity. These waveguide studies used type-0 interaction and QPM to reduce the residual phase mismatch and thereby increase $n_{2, \text { casc }}$. However, it was recently shown [7] that QPM is often detrimental because the cascaded quadratic nonlinear response can become resonant [10], implying a narrow-bandwidth nonlinearity that impedes nonlinear optics on the few-cycle scale. Moreover, reducing the effective $\Delta k$ also gives a much stronger cascading-induced pulse self-steepening [11]. In other words, using QPM in tailoring the cascaded quadratic nonlinearity is a twoedged sword; even if it may increase the cascading nonlinearity it will also lead to detrimental nonlinear effects.

In this Letter, we show that, without a QPM structure, a commercial LN ridge waveguide could also have a nonresonant effective self-defocusing nonlinear index, and supports the formation of self-compressed few-cycle solitons in the near-IR. This is achieved through a dominant negative cascaded quadratic nonlinearity (see the diagram in Fig. 1). The waveguide is based on the direct bonding of a $\mathrm{LN}$ layer to a lithium tantalate (LT) substrate and then is diced to have a ridge profile $[12,13]$. Since the LN core and the LT substrate have similar effective refractive index (RI), the waveguide dispersive phase (mode propagation constant) is restricted to be close to the material phase, which makes the phase-mismatch 


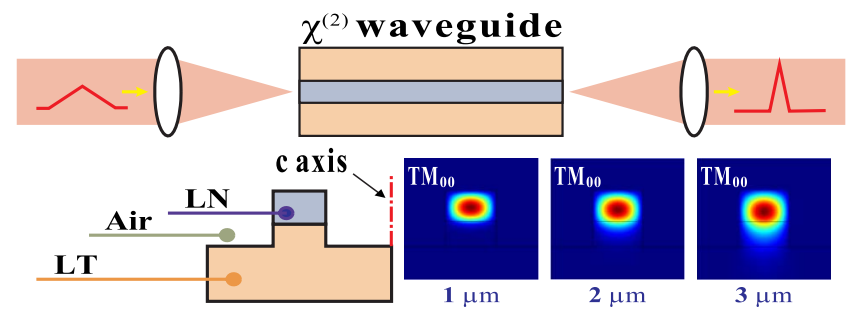

Fig. 1. Diagram of cascaded quadratic soliton compression in a $\mathrm{LN}$ ridge waveguide. Fundamental mode $\left(\mathrm{TM}_{00}\right)$ distributions at different wavelengths $(1,2$, and $3 \mu \mathrm{m})$ are also shown, with structure marked as S-1. $c$ axis is the optic axis of the crystal, i.e., the crystallographic $z$-axis.

profile as well as dispersion profile almost unchanged. With the large quadratic susceptibility inherited from the material, a strong cascaded quadratic nonlinearity in such a QPM-free waveguide is possible through a noncritical type-0 SHG process, which exploits the largest quadratic nonlinear tensor component $d_{33}$, and importantly is inherently strongly phase-mismatched $(\Delta k$ large and positive) leading automatically to a self-defocusing cascading nonlinearity. If this becomes strong enough to outbalance the self-focusing material Kerr nonlinearity, excitation of temporal solitons is possible within the normal dispersion region.

The dispersion properties of the LN ridge waveguide are calculated through eigenmodes of the waveguide, including both mode distributions $U$ and propagation constants $\beta$, by using the software COMSOL Multiphysics. Examples of fundamental $\mathrm{TM}_{00}$ mode distributions at different wavelengths are shown in Fig. $\underline{1}$. In Fig. $\underline{2(a)}$ we

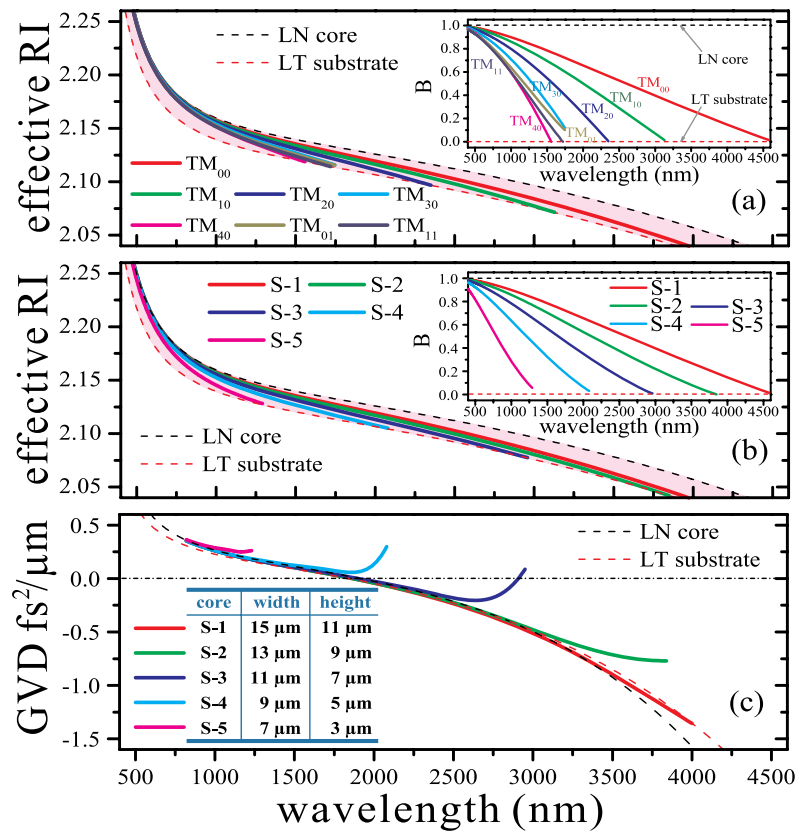

Fig. 2. Dispersion properties of the LN ridge waveguide. (a) Effective RIs of eigenmodes, in the waveguide structure S-1. (b) Fundamental mode effective RIs of different waveguide structures; inserts in (a) and (b) show normalized effective RIs in which the material dispersion is removed. (c) GVD profiles of the fundamental mode in different waveguide structures. Detailed waveguide sizes are shown as insert. show effective RIs of extraordinary eigenmodes (with the electric field polarized along the crystallographic $z$-axis). As guided modes, they should have the effective RI lying between the substrate RI and the core RI. Since the LN core and the LT substrate have small RI difference, the confinement of the waveguide is quite weak and the eigenmodes are restricted to a narrow strip of the effective RIs, which results in two consequences: (1) the mode effective RIs and mode cutoff wavelengths will become sensitive to the waveguide (core) size; (2) the effective RI profiles (especially for the fundamental $\mathrm{TM}_{00}$ mode) as well as the dispersion properties remain close to the material profile as there is little room for variation.

A normalized propagation constant is defined to help remove the material dispersion and to better illustrate the eigenmodes in the waveguide: $B=\left(n_{\text {eff }}^{2}-n_{\text {sub }}^{2}\right) /$ $\left(n_{\text {core }}^{2}-n_{\text {sub }}^{2}\right)$ [14], with $n_{\text {eff }}, n_{\text {core }}$, and $n_{\text {sub }}$ representing the mode effective RI, the LN core RI, and the LT substrate RI, respectively. Figure 2(b) shows that with a decreasing core size the fundamental mode is strongly impacted and its cutoff wavelength is shortened as the waveguide confinement is further reduced. Meanwhile, the group velocity dispersion (GVD coefficient $\beta^{(2)}=$ $d^{2} \beta / d \omega^{2}$ ) profiles tend to follow the material profile except for a deflection around the cutoff wavelength, see Fig. 2(c), as there the effective RI is approaching and turning into the substrate RI and a large waveguide dispersion is produced. For structures S-1, S-2 and S-3, the fundamental mode will have both normal and anomalous GVD regions, transiting at a single zero dispersion wavelength (ZDW), which is close to the material $\mathrm{ZDW}$ around $1900 \mathrm{~nm}$. Fundamental modes in structures S-4 and S-5 have very short cutoff wavelengths so that they just have normal GVD regions.

We now estimate the nonlinearities in the waveguide. The relevant cascaded nonlinearity is represented as the nonlinear coefficient $\gamma_{\text {casc }}=(\omega / c)\left(n_{2, \text { casc }} / A_{\text {eff,casc }}\right)[\underline{15}$ ], which is analogous to the material Kerr nonlinearity scaled as $\gamma_{\text {Kerr }}=(\omega / c)\left(n_{2, \text { Kerr }} / A_{\text {eff,Kerr }}\right)$ [16], where $A_{\text {eff,casc }}$ and $A_{\text {eff,Kerr }}$ are effective mode areas corresponding to the cascaded nonlinearity and the Kerr nonlinearity, respectively. It should be noted that $A_{\text {eff,casc }}$ actually stems from a SHG process and therefore has a different definition to the commonly known $A_{\text {eff,Kerr }}$ from a Kerr SPM process. A dispersive $A_{\text {eff.casc }}$ referring to the SHG within the $\mathrm{TM}_{00}$ mode is defined as [15]

$$
\begin{aligned}
& A_{\text {eff,casc }}\left(\omega_{1}\right) \\
& \quad=\frac{\left(\iint \mathrm{d} x \mathrm{~d} y\left|U_{\mathrm{TM}_{00}}\left(\omega_{2}\right)\right|^{2}\right)\left(\iint \mathrm{d} x \mathrm{~d} y\left|U_{\mathrm{TM}_{00}}\left(\omega_{1}\right)\right|^{2}\right)^{2}}{\left(\iint \mathrm{d} x \mathrm{~d} y U_{\mathrm{TM}_{00}}^{*}\left(\omega_{2}\right) U_{\mathrm{TM}_{00}}^{2}\left(\omega_{1}\right)\right)^{2}},
\end{aligned}
$$

where $U_{\mathrm{TM}_{00}}$ is the eigenmode distribution of the $\mathrm{TM}_{00}$ mode, $\omega_{1}$ and $\omega_{2}$ are angular frequencies of the pump and the SH. $A_{\text {eff,casc }}$ as a function of the pump wavelength is illustrated in Fig. 3(a), which is quite close to the $A_{\text {eff,Kerr }}$ referring to the Kerr SPM process within the $\mathrm{TM}_{00}$ mode. Other effective mode areas stemming from interactions among high-order modes (also phasemismatched) are much larger than the two shown due 


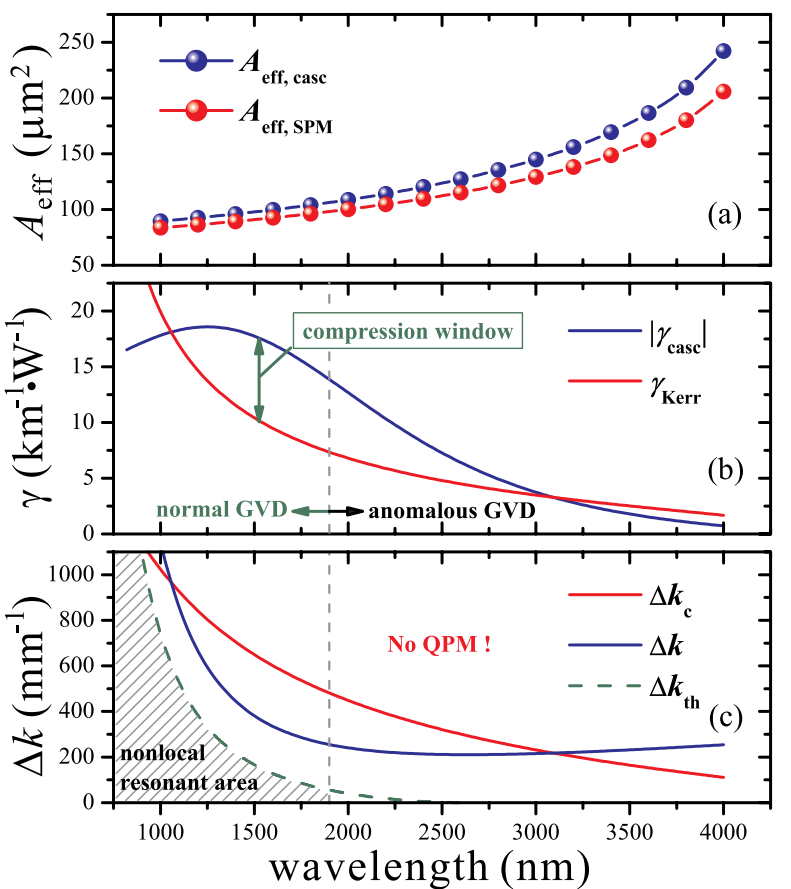

Fig. 3. Nonlinearities in the LN ridge waveguide with structure S-1. (a) Effective mode area corresponding to the cascaded SHG process and Kerr SPM process in the fundamental mode $\mathrm{TM}_{00}$. (b) The nonlinear factor of the cascaded quadratic nonlinearity as well as the Kerr nonlinearity, a compression window is shown from 1100 to $1900 \mathrm{~nm}$ in which a dominant self-defocusing nonlinearity is achieved. (c) Equivalently, the phase mismatch is below the critical value $\Delta k_{c}$ marking the onset of a self-focusing nonlinearity, and it is nonresonant as it stays above the nonlocal resonant area (marked area).

to the orthogonality among different modes, and the corresponding nonlinear factors $\gamma$ are therefore largely reduced and negligible.

Figure 3(b) shows that the negative nonlinear cascading factor $\gamma_{\text {casc }}$ for the $\mathrm{TM}_{00}$ mode is stronger than the Kerr nonlinear factor $\gamma_{\text {Kerr }}$ over a broad wavelength span $(1100-3000 \mathrm{~nm})$. Such a broadband self-defocusing nonlinearity is actually built up due to the large susceptibility $d_{33}$ of the LN material, which gives rise to a dominant $n_{2, \text { casc }}$ [7]. However, such a self-defocusing nonlinearity should work with the normal GVD to excite solitons, so the window of operation is actually from $1100 \mathrm{~nm}$ to the GVD transition position at $1900 \mathrm{~nm}$. Within such a "compression window" [17] the phase-mismatch parameter is actually below the critical value $\Delta k_{c}$ referring the balance between the cascaded and the Kerr nonlinearity, see Fig. 3(c). Meanwhile, the phase mismatch is larger than the threshold $\Delta k_{\text {th }}$ to the resonant regime, which means the cascaded response is ultrafast, broadband, and without the characteristic resonant spectral peaks generated if $\Delta k$ is in the marked area $[10,18,19]$. Therefore, we may expect few-cycle soliton formation with high pulse quality. Unlike most LN waveguides having QPM to tune the phase mismatch, the presented LN ridge waveguide is actually QPM-free as it is naturally suitable for few-cycle soliton formation, just like the bulk LN case investigated in [7].

Summarizing, the total nonlinearity governing the pulse SPM is $\gamma_{\text {eff }}=\gamma_{\text {casc }}+\gamma_{\text {Kerr }}$. The nonlinear length of the waveguide is then calculated as $L_{N}=\left|P \cdot \gamma_{\mathrm{eff}}\right|^{-1}$, where $P$ is the peak power of the pump pulse. Furthermore, with the dispersion length $L_{D}=T_{0}^{2} /\left|\beta^{(2)}\right|\left(T_{0}\right.$ is the pulse duration), the soliton number $N$ can be finally estimated as $N^{2}=L_{D} / L_{N}$.

Now we numerically investigate the few-cycle soliton formation in an LN ridge waveguide. The numerical model is the nonlinear wave equation in frequency domain (NWEF), which accurately models ultrashort pulse propagation in nonlinear materials [19]. In waveguides, the NWEF is actually governing the propagation dynamics of the pulse electric field amplitude while the pulse transverse distribution is described in form of waveguide eigenmode distributions [20].

We first show pumping at $1550 \mathrm{~nm}$ where the total selfdefocusing nonlinearity is maximum. The input pulse has 10-nJ energy, 50-fs FWHM, and it is in the $\mathrm{TM}_{00}$ mode. During the propagation, the pulse spectrum evolves with SPM-induced spectral broadening. Meanwhile, a solitoninduced optical Cherenkov wave (or dispersive wave, DW) [21] is generated at opposite GVD region around $3000 \mathrm{~nm}$ due to the perturbation of higher-order dispersion, see Figs. 4(a) and 4(b). Combined with the normal dispersion, the ultrabroad spectrum leads to the formation of a single-cycle self-compressed soliton. In Fig. 4(c), the pulse profile with significant compression while its peak power is enhanced over 6 times. After the compression, the pulse is split into several branches due to the Raman fraction ( $(50 \%)$ in the $\mathrm{LN}$ material, known as the Raman-induced pulse splitting [22]. Figure 4(d) shows the electric field amplitude of the compressed pulse, with clean and single-cycle profile. The quality factor (defined in [17]) is around 0.4. Actually,

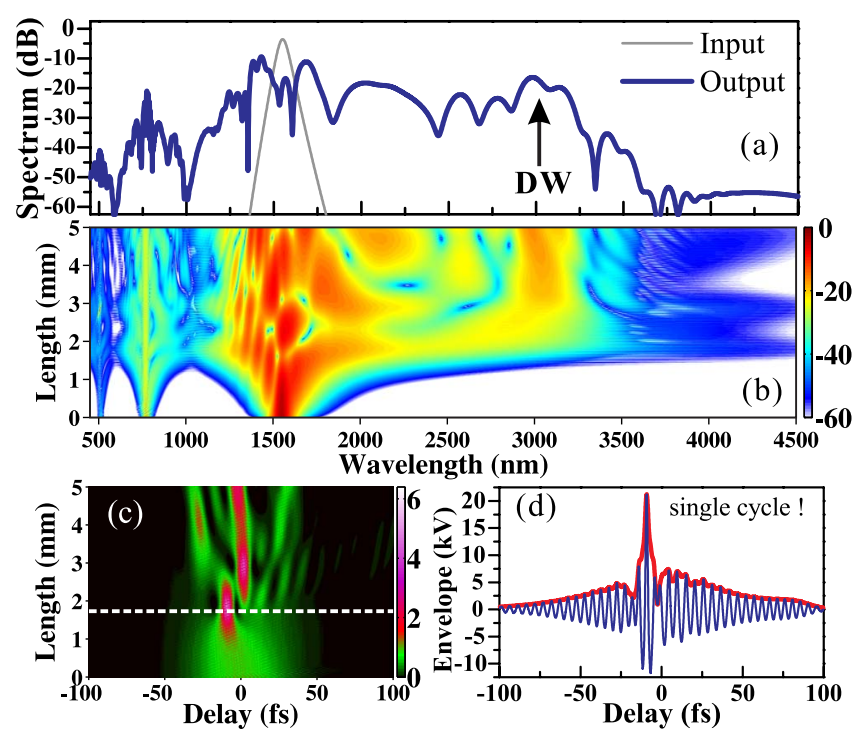

Fig. 4. Numerical simulation of soliton self-compression in the $\mathrm{LN}$ ridge waveguide with structure S-1, pumped at $1550 \mathrm{~nm}$. The pump pulse has a FWHM of $50 \mathrm{fs}, P=200 \mathrm{~kW}$ giving a pulse energy of $10 \mathrm{~nJ} ; \gamma_{\text {casc }}=-17.6 \mathrm{~km}^{-1} \mathrm{~W}^{-1}$ and $\gamma_{\text {Kerr }}=$ $9.9 \mathrm{~km}^{-1} \mathrm{~W}^{-1}$, giving $N=3.7$. (a) Pulse spectrum at the input and the output; (b) pulse spectral evolution (in $\mathrm{dB}$ scale); (c) pulse temporal evolution with the self-compression position marked by the white dashed line (scaled to the peak power of the input pulse); (d) physical real-valued electric field amplitude of the self-compressed pulse. 


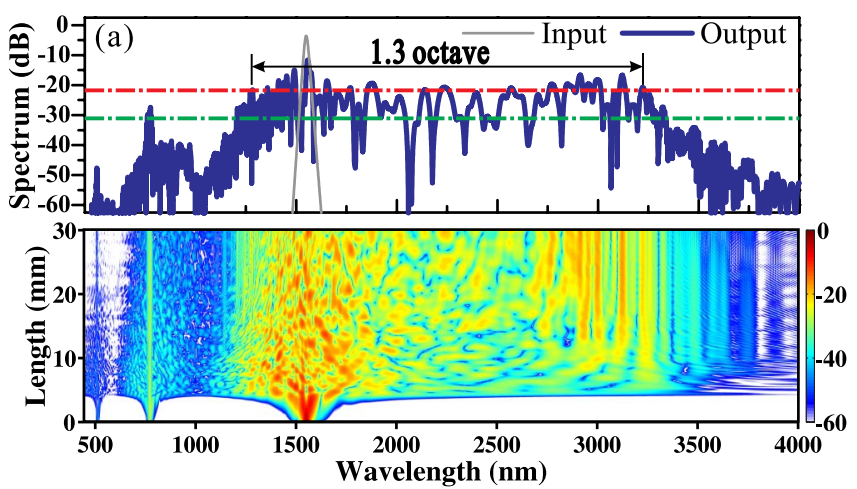

Fig. 5. Supercontinuum generation in the waveguide S-1 pumped at $1550 \mathrm{~nm}$. The pump pulse has 30-nJ energy, 150fs FWHM; the soliton order is $N=11.1$. (a) Pulse supercontinuum spectrum at the input and the output (the red dashed line marks the $-10 \mathrm{~dB}$ range of the output spectrum, the green dashed line marks the $-20 \mathrm{~dB}$ range); (b) pulse spectral evolution.

at other wavelengths and in the other structures, S2 to S5, such scenario of the cascaded quadratic soliton compression also works only if the pump pulse is within the compression window as illustrated above.

Aside from the soliton compression, such a waveguide can also be applied for SCG. By launching a longer pulse with more energy, namely 150-fs FWHM and 30-nJ energy, the soliton number is increased correspondingly to 11.10 and a supercontinuum spectrum covering $1300-3200 \mathrm{~nm}$ with 1.3 octave bandwidth (at $-10 \mathrm{~dB}$ ) is accomplished, as shown in Fig. 5. As opposed to SCG in photonic crystal fibers [23], here the combination of self-defocusing nonlinearities and soliton formation in the normal dispersion regime leads to the blue edge of the spectrum being supported by cascading-induced pulse self-steepening [11], while the red-edge formation occurs due to the Raman-induced pulse splitting of the solitons in the nonlinear regime, and soliton-induced DW generation in the linear long-wavelength regime of anomalous dispersion [21].

In summary, we investigated a commercial LN ridge waveguide structure and found that strong cascaded quadratic nonlinearity could be produced through the QPM-free type-0 SHG process in the waveguide. Therefore, the waveguide can be applied for cascaded quadratic soliton compression, which makes use of the selfdefocusing nonlinearity combined with normal dispersion. By carefully studying the dispersion properties and the nonlinearities in such a QPM-free waveguide, the compression window is concluded to be from 1100 to $1900 \mathrm{~nm}$, which exactly covers the whole communication band. At $1550 \mathrm{~nm}$, single cycle pulse compression was observed by pumping a low-energy pulse, while when a longer pulse is launched, the waveguide can produce octave-spanning SCG. With compact size, simple structure and high nonlinearities, such a QPM-free quadratic waveguide could have great potential in optical communications and laser physics.

M. B. and B. Z. acknowledge support from the Danish Council for Independent Research (274-08-0479, 11106702). X. L. Z. acknowledges support from a Marie Curie fellowship (COPULCO: 253289), the National Natural Science Foundation of China (11274224, 60978004), and Shanghai Shuguang Program (10SG38).

\section{References}

1. O. Tadanaga, T. Yanagawa, Y. Nishida, H. Miyazawa, K. Magari, M. Asobe, and H. Suzuki, Appl. Phys. Lett. 88, 061101 (2006).

2. M. Levenius, V. Pasiskevicius, F. Laurell, and K. Gallo, Opt. Express 19, 4121 (2011).

3. R. DeSalvo, D. J. Hagan, M. Sheik-Bahae, G. Stegeman, E. W. Van Stryland, and H. Vanherzeele, Opt. Lett. 17, 28 (1992).

4. X. Liu, L.-J. Qian, and F. W. Wise, Opt. Lett. 24, 1777 (1999).

5. S. Ashihara, J. Nishina, T. Shimura, and K. Kuroda, J. Opt. Soc. Am. B 19, 2505 (2002).

6. J. Moses and F. W. Wise, Opt. Lett. 31, 1881 (2006).

7. B. Zhou, A. Chong, F. Wise, and M. Bache, Phys. Rev. Lett. 109, 043902 (2012).

8. C. Langrock, M. Fejer, I. Hartl, and M. E. Fermann, Opt. Lett. 32, 2478 (2007).

9. C. Phillips, C. Langrock, J. Pelc, M. Fejer, J. Jiang, M. E. Fermann, and I. Hartl, Opt. Lett. 36, 3912 (2011).

10. M. Bache, O. Bang, J. Moses, and F. W. Wise, Opt. Lett. 32 , 2490 (2007).

11. J. Moses and F. W. Wise, Phys. Rev. Lett. 97, 073903 (2006).

12. M. Asobe, Y. Nishida, O. Tadanaga, H. Miyazawa, and H. Suzuki, IEICE Trans. Electron. E88-C, 335 (2005).

13. T. Umeki, O. Tadanaga, and M. Asobe, IEEE J. Quantum Electron. 46, 1206 (2010).

14. K. Okamoto, Fundamentals of Optical Waveguides (Academic, 2010).

15. M. Bache, J. Opt. Soc. Am. B 26, 460 (2009).

16. G. Agrawal, Nonlinear Fiber Optics (Academic, 2013).

17. M. Bache, J. Moses, and F. W. Wise, J. Opt. Soc. Am. B 24, 2752 (2007).

18. M. Bache, O. Bang, W. Krolikowski, J. Moses, and F. W. Wise, Opt. Express 16, 3273 (2008).

19. H. Guo, X. Zeng, B. Zhou, and M. Bache, J. Opt. Soc. Am. B 30, 494 (2013).

20. H. Guo, X. Zeng, and M. Bache, "Generalized nonlinear wave equation in frequency domain," arXiv:1301.1473 (2013).

21. M. Bache, O. Bang, B. B. Zhou, J. Moses, and F. W. Wise, Phys. Rev. A 82, 063806 (2010).

22. K. Tai, N. Bekki, and A. Hasegawa, Opt. Lett. 13, 392 (1988).

23. J. Dudley, G. Genty, and S. Coen, Rev. Mod. Phys. 78, 1135 (2006). 